\title{
Fatty acid activation in carcinogenesis and cancer development: Essential roles of long-chain acyl-CoA synthetases (Review)
}

\author{
YUE TANG $^{1,2}$, JING ZHOU ${ }^{3}$, SHING CHUAN HOOI ${ }^{4}$, YUE-MING JIANG ${ }^{1 *}$ and GUO-DONG LU ${ }^{1,2,5^{*}}$ \\ ${ }^{1}$ Department of Toxicology, School of Public Health, Guangxi Medical University; ${ }^{2}$ Guangxi Colleges and Universities \\ Key Laboratory of Prevention and Control of Highly Prevalent Diseases, Guangxi Medical University; ${ }^{3}$ Department of \\ Physiology, School of Preclinical Medicine, Guangxi Medical University, Nanning, Guangxi 530021, P.R. China; \\ ${ }^{4}$ Department of Physiology, Yong Loo Lim School of Medicine, National University of Singapore, Singapore 117543, \\ Republic of Singapore; ${ }^{5}$ Key Laboratory of High-Incidence-Tumor Prevention and Treatment, Guangxi \\ Medical University, Ministry of Education of China, Nanning, Guangxi 530021, P.R. China
}

Received January 21, 2018; Accepted May 22, 2018

DOI: $10.3892 / \mathrm{ol} .2018 .8843$

\begin{abstract}
The significance of fatty acid metabolism in cancer initiation and development is increasingly accepted by scientists and the public due to the high prevalence of overweight and obese individuals. Fatty acids have different turnovers in the body: Either breakdown into acetyl-CoA to aid ATP generation through catabolic metabolism or incorporation into triacylglycerol and phospholipid through anabolic metabolism. However, these two distinct pathways require a common initial step known as fatty acid activation. Long-chain acyl-CoA synthetases (ACSLs), which are responsible for activation of the most abundant long-chain fatty acids, are commonly deregulated in cancer. This deregulation is also associated with poor survival in patients with cancer. Fatty acids physiologically regulate ACSL expression, but cancer cells could hijack certain involved regulatory mechanisms to deregulate ACSLs. Among the five family isoforms, ACSL1 and ACSL4 are able to promote ungoverned cell growth, facilitate tumor invasion and evade programmed cell death, while ACSL3 may have relatively complex functions in different types of cancer. Notably, ACSL4 is also essential for the induction of ferroptosis (another form of programmed cell death) by facilitating arachidonic acid oxidation, which makes the enzyme a desirable cancer target. The present review thus evaluates the functions of deregulated ACSLs in cancer, the possible
\end{abstract}

Correspondence to: Dr Yue-Ming Jiang or Dr Guo-Dong Lu, Department of Toxicology, School of Public Health, Guangxi Medical University, 22 Shuangyong Road, Nanning, Guangxi 530021, P.R. China

E-mail: ymjianggxmu@163.com

E-mail: golden_lu@hotmail.com

${ }^{*}$ Contributed equally

Key words: long-chain acyl-CoA synthetases, carcinogenesis, cancer development, cell death, fatty acids, metabolism molecular mechanisms involved and the chemotherapeutic potentials to target ACSLs. A better understanding of the pathological effects of ACSLs in cancer and the involved molecular mechanisms will aid in delineating the exact role of fatty acid metabolism in cancer and designing precise cancer prevention and treatment strategies.

\section{Contents}

1. Introduction

2. Subcellular localization and physiological functions of ACSLs

3. Deregulated expression of ACSLs in clinical cancer

4. Molecular mechanisms to deregulate ACSL expression in cancer

5. Effects of ACSLs on carcinogenesis and cancer development

6. Conclusions

\section{Introduction}

Fatty acids are essential nutrients, either obtained externally from daily meals or derived internally from de novo synthesis and breakdown of cellular triacylglycerol (TAG) and phospholipid. As important building blocks of the body, they also participate in energy metabolism and cellular signaling pathways to maintain physiological functions. However, deregulated fatty acid metabolism favoring excess lipid biosynthesis and deposition eventually predisposes the body to metabolic disorders and carcinogenesis.

Fatty acids have different turnovers in the body (Fig. 1). They can break down through a series of mitochondrial $\beta$-oxidation processes into acetyl-CoA, which then enters the tricarboxylic acid cycle to aid ATP generation. Alternatively, fatty acids can be incorporated into TAG, phospholipids or cholesterol esters. It is noteworthy that the two distinct pathways require a common initial step known as fatty acid activation by acyl-CoA synthetase (ACS) $(1,2)$. Long-chain ASCs (ACSLs) are essential enzymes for the activation of the 
most abundant long-chain fatty acids (12-20 carbons) $(2,3)$. By contrast, fatty acids with $<6$ carbons (e.g., acetate, propionate and butyrate) are activated by short-chain ACSs (ACSS) and fatty acids with 6-10 carbons are activated by medium-chain ACSs, while very long-chain fatty acids (>20 carbons) are activated by very long-chain ACSs (1-3).

Five isoforms of ACSLs, namely ACSL1, ACSL3, ACSL4, ACSL5 and ACSL6, are present in mammals, and they have overlapping but specific roles in the activation of long-chain fatty acids. Individual ACSL isoforms may have preferred substrates for activation, dependent on the chain length and saturation status of fatty acids $(1,2)$. For example, ACSL3 and ACSL4 can activate polyunsaturated fatty acids (PUFA), but ACSL3 prefers oleic acid, whereas ACSL4 favors arachidonic acid (AA) and adrenic acid $(1,2)$.

Recent pathological studies have found the abnormal expression of ACSLs in cancer tissues in comparison to neighboring non-cancerous tissues $(2,3)$, as discussed below. Furthermore, their oncogenic roles and certain involved molecular mechanisms have been unveiled. The present review thus focuses on ACSLs, evaluating the recent evidence of the pathological functions of ACSLs in carcinogenesis and cancer development, and discussing the chemotherapeutic potential of targeting ACSLs.

\section{Subcellular localization and physiological functions of ACSLs}

ACSL-mediated fatty acid activation contributes to the two distinct pathways of anabolic lipid biosynthesis and catabolic fatty acid oxidation. The manner in which cells adapt to channel fatty acids into the distinct anabolic or catabolic pathways is currently poorly understood. Certain early hypotheses suggested that the subcellular localization of ACSLs and specified fatty acid transportation system may contribute to channeling fatty acids into different turnovers $(1,2,4)$. Fig. 2 depicts a mitochondrion, where ACSL1, ACSL4 and ACSL5 localize and support fatty acid synthesis and $\beta$-oxidation, and a peroxisome, where ACSL1 and ACSL4 are involved in alkyl lipid biosynthesis and $\beta$-oxidation. ACSL1, ACSL3 and ACSL4 have also been found to reside in the endoplasmic reticulum (ER), facilitating glycerolipid synthesis and $\omega$-oxidation (a minor pathway for medium-chain fatty acids in the normal physiological condition, but an alternative pathway when $\beta$-oxidation is defective). In addition, ACSL3 in lipid droplets may aid neutral lipid synthesis and lipid droplet formation (5).

Tissue-specific loss-of-function studies are particularly useful to unveil the physiological function of individual ACSL isoforms. The majority of studies have focused on ACSL1, the founding member of the ACSL family. Liver-specific knockout of ACSL1 reduced TAG synthesis and fatty acid oxidation (6). In contrast, adipose-specific (7), skeletal muscle-specific (8) or heart-specific (9) knockout of ACSL1 exhibited common phenotypes of decreased fatty acid oxidation alone. In addition, transgenic mice with all-tissue ACSL5-knockout exhibited decreased adiposity and increased energy expenditure, indicating its major role in fatty acid biosynthesis and deposition (10). Thus, the specific role of ACSL5 may be different from that of ACSL1. However, for the remaining ACSL isoforms, there are no such loss-of-function mouse models available.

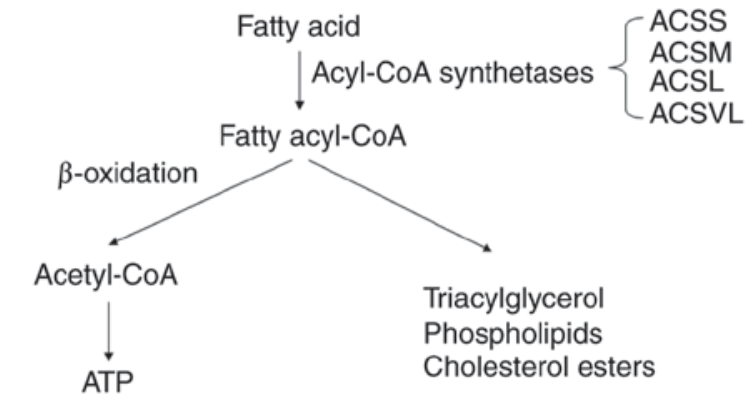

Catabolic metabolism

Anabolic metabolism

Figure 1. Acyl-CoA synthetases-mediated fatty acid activation is involved in two distinct lipid metabolism pathways. ACSS, short-chain acyl-CoA synthetase; ACSM, medium-chain acyl-CoA synthetase; ACSL, long-chain acyl-CoA synthetase; ACSVL, very long-chain acyl-CoA synthetase.

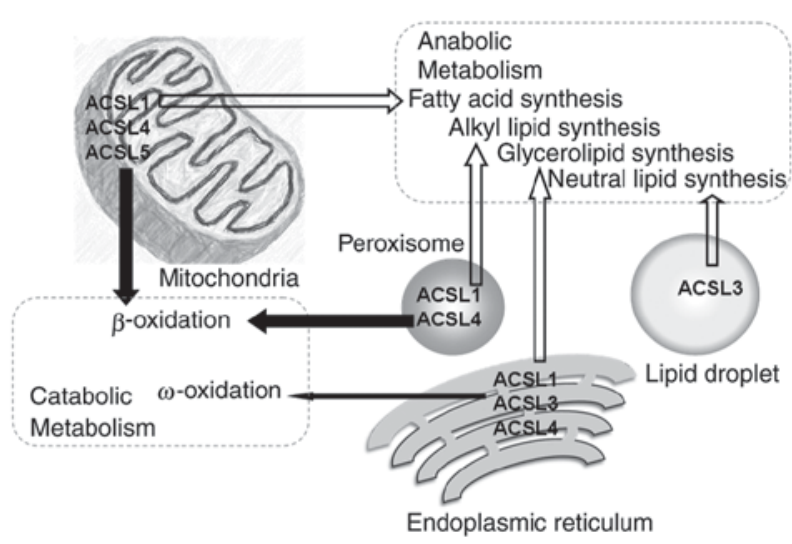

Figure 2. Subcullular localization and function of ACSL isoforms. ACSL members reside in different subcellular organelles to channel fatty acids into two distinct metabolic pathways. The white arrows refer to channeling to anabolic metabolism, while the black arrows refer to catabolic metabolism. ACSL, long-chain acyl-CoA synthetase.

Taken together, these results suggest that ACSLs are important for fatty acid metabolism. Whether individual ACSL isoforms channel fatty acids toward anabolic metabolism or catabolic metabolism may be dependent on their subcellular localization and interaction with specific fatty acid transportation systems. It is also possible that certain compositions of fatty acids could affect the expression and localization of ACSL isoforms in physiological and pathological conditions.

\section{Deregulated expression of ACSLs in clinical cancer}

Accumulating findings have demonstrated that almost all ACSL members are deregulated in clinical cancer and that a number are associated with poor patient survival. The expressional changes of ASCLs in different types of cancer are summarized in Fig. 3, with the asterisk marks indicating statistically significant associations with patient prognosis. Upregulation of ACSL1 was found in multiple types of cancer, including colon $(11,12)$, breast $(13)$ and liver $(14,15)$ cancer, and myeloma (16), while downregulation was found in lung squamous cell carcinoma (17). More importantly, ACSL1 overexpression was associated with a poor clinical outcome in colon cancer patients (18). Like those of ACSL1, the majority 


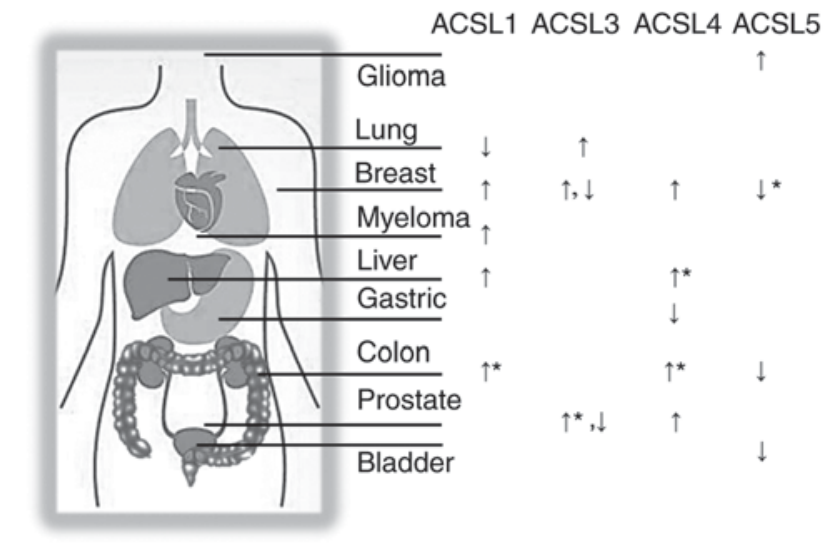

Figure 3. Deregulated ACSL expression in cancer. The expressional changes of individual ACSL isoforms in different types of cancer were summarized. The asterisk indicates statistically significant associations with poor cancer survival outcomes. ACSL, long-chain acyl-CoA synthetase.

of studies of ACLS4 favor an oncogenic role. ACSL4 has been shown to be overexpressed in multiple cancer types, including colon $(11,19)$, breast $(20)$, liver (21-23) and prostate (24) cancer, while another study has shown its downregulation in gastric cancer (25). In terms of patient survival outcome, ACSL4 overexpression predicted poorer patient survival in stage II colon cancer [together with upregulated stearoyl-CoA desaturase (SCD)] (12) and in liver cancer (together with downregulated growth arrest and DNA damage inducible $\beta$ ) (21).

By contrast, ACSL5 appears to exhibit the opposite function in cancer. ACSL5 was downregulated in colon (26) and breast (27) cancer, where ACSL1 and ACSL4 were upregulated. ACSL5 was also decreased in bladder cancer (28). More importantly, lower expression of ACSL5 in breast cancer was associated with a worse prognosis (27). An exception was reported in glioma, where ACSL5 was upregulated (29). One recent study also demonstrated that fibroblast growth factor receptor 2 (FGFR2)-ACSL5 chimera RNA rendered clinical gastric cancer cells resistant to treatment with FGFR inhibitors (30).

The role of ACSL3 in cancer is comparatively complex. ACSL3 was found to be overexpressed in lung cancer (31), prostate cancer (32) and estrogen receptor-negative breast cancer (33). Furthermore, its upregulation predicted an unfavorable prognosis in prostate cancer (32). However, opposing results were also determined in studies conducted in prostate cancer and breast cancer. The expression of ACSL3 was decreased in high-grade and metastatic prostate cancer (34). Homozygous deletion of ACSL3 was found in breast cancer following chemotherapy, and was associated with increased risks of recurrence and distant metastasis (35). These contradicting results may suggest that the roles of ACSL3 vary among the different stages of cancer. Lastly, few studies have reported on ACSL6 in cancer. Only one case report found a t $(5 ; 12)(q 23-31 ; p 13) / E T V 6-A C S L 6$ gene fusion in chronic leukemia, which rendered cancer cells resistant to treatment using a tyrosine kinase inhibitor (36).

A recent bioinformatics study (37) utilizing online databases Oncomine (https://www.oncomine.org/resource/login .html) and PrognoScan (http://www.abren.net/PrognoScan/) stated certain novel associations between ACSL expression and cancer survival outcomes. On the one hand, which was in line with previous findings $(12,18,27)$, the study showed that the overexpression of either ACSL1 or ACSL4 was associated with a poorer prognosis in patients with colon cancer, and that ACSL5 downregulation was associated with poor survival in breast cancer patients. On the other hand, the study suggested certain novel links. High ACSL1 expression was associated with worse survival in lung cancer patients and high ACSL3 was associated with worse survival in patients with melanoma. However, there are also opposing results (37) linking higher ACSL expression with improved patient survival in multiple types of cancer, including breast, ovarian, brain and lung cancer, and acute myeloid leukemia. The dry-lab bioinformatic approach enabled fast analyses and novel associative findings, but the obtained results may require further validations using datasets from the other databases or sources.

Taken together, the results from the majority of studies supported the fact that ACSL1 and ACSL4 serve oncogenic roles in the majority of cancer types, whereas ACSL5 may suppress tumor development. However, the function of ACSL3 in cancer may be dependent on the stages of prostate and breast cancer. The aforementioned ACSL-knockout transgenic mice models, alone or cross-bred with other transgenic mice with known oncogenes [e.g., KRAS proto-oncogene, GTPase and $\mathrm{HBx}$ (hepatitis B virus X-interacting protein), etc.], will be useful to shed light on the cause-effect function of ACSLs in carcinogenesis and cancer development.

\section{Molecular mechanisms to deregulate ACSL expression in cancer}

ACSLs serve essential roles in fatty acid activation and metabolism, and their expression is also readily controllable by intracellular fatty acids in physiological and pathological conditions. Physiologically, diet-derived fatty acids can activate several transcription factors, including peroxisome proliferator-activated receptors $\alpha$ and $\delta$ (PPAR $\alpha$ and PPAR $\delta$ ), liver $X$ receptors $\alpha$ and $\beta$ (LXR $\alpha$ and LXR $\beta$ ), cAMP response element-binding and specificity protein 1 (Sp1) $(13,15,38,39)$. These transcription factors can then activate different ACSLs through direct binding to conserved responsive elements in the ACSL promoter regions. Pathologically, cancer cells could hijack these transcriptional mechanisms to upregulate ACSLs, as evidenced by the following three examples. Firstly, cancer cells can utilize an epigenetic approach to activate ACSL indirectly. PPAR $\alpha$ increases ACSL1 transcription, but this action could be repressed by microRNA-9 (miR-9) in the normal liver. However, liver cancer overexpressed the long non-coding RNA (lncRNA) known as highly upregulated in liver cancer. This lncRNA could dampen miR-9 and relieve its suppression of PPAR $\alpha$ expression and consequently turn on ACSL1 transcription (15). Secondly, oncogenes can activate ACSL transcription indirectly through modulation of the aforementioned transcription factor. In breast cancer cells, HBx was reported to act as a co-activator to sensitize Sp1-mediated transcription of ACSL1 (13). Thirdly, hepatitis B virus mutant large surface protein also increased ACSL3 expression through induction of ER stress, and this action could promote lipid biosynthesis and deposition (40). Glycogen synthase kinase $3 \beta$ can also activate ACSL3 in an ER stress-dependent manner, but the exact mechanism remains unclear. 
Apart from direct regulation of ACSL transcription, cancer cells can manipulate the mRNA stability of ACSLs by targeting their $3^{\prime}$-untranslated regions (3'UTR). Previous studies reported that miR-205 was downregulated in liver cancer (41) and lung squamous cell carcinoma (17) to augment ACSL1 mRNA stability, since miR-205 can directly bind to the 3'UTR of ACSL1 and facilitate its degradation. A recent study further showed that DNA polymorphism (rs8086, T/T genotype) in the 3'UTR of the ACSL1 gene enabled higher ACSL1 expression compared with the corresponding $\mathrm{C} / \mathrm{T}$ or $\mathrm{C} / \mathrm{C}$ genotype. It was suggested that the genotype difference in 3'UTR may affect the binding affinity of microRNA and the resultant ACSL1 mRNA stability. More importantly, those patients with the $\mathrm{T} / \mathrm{T}$ genotype and colon cancer had a poor disease-free survival outcome (18). Note that, in liver cancer, HBx repressed miR-205 expression and consequently increased ACSL4 mRNA stability (41). It is thus rational to propose that HBx can activate ACSL1 using the same mechanism.

Alternatively, protein ubiquitination and the involved proteasome degradation pathway is another mechanism to regulate ACSL expression. Fatty acid AA treatment could enhance ACSL4 protein ubiquitination and shorten its protein half-life in HepG2 cells via a negative feedback loop (42), as AA is the preferred substrate of ACSL4. By contrast, in breast cancer, the hormone 17 $\beta$-estradiol extended the half-life of the ACSL4 protein, and subsequently increased cellular uptake of AA and eicosapentaenoic acid (43). This 17 $\beta$-estradiol-mediated ACSL4 upregulation was found to be essential for the invasiveness of estrogen receptor-expressing breast cancer cells.

\section{Effects of ACSLs on carcinogenesis and cancer development}

ACSL-mediated lipid anabolism may promote cancer initiation. Silencing of ACSL3 markedly inhibited HCV secretion, suggesting that phospholipid generation by the ACSL pathway is involved in the replication and secretion of the hepatitis B virus and the hepatitis $C$ virus (44). The latter two viruses are well-known etiologies of liver cancer. Note that cancer is characterized by 10 hallmarks, including self-sufficiency in growth signals, deregulated metabolism, tissue invasion and metastasis, and evasion of programmed cell death (45). Next, the effects of ACSLs on the relevant cancer hallmarks and the possible molecular mechanisms involved are summarized.

ACSLs and ungoverned cell proliferation. Fatty acids promote unchecked cancer cell proliferation directly, by providing essential biosynthetic and functional intermediates. Cholesterol supplementation alone enhanced cell proliferation in liver cancer cells (41). Several studies highlighted that ACSLs stimulated proliferation in cancer cells. Knockdown of ACSL1, ACSL3 or ACSL4 independently decreased cell proliferation and anchorage-independent growth in multiple cancer cells and xenograft tumor growth in nude mice $(11,21,31,43)$. By contrast, forced overexpression of these ACSLs increased cell proliferation and tumor growth $(15,20,24,46,47)$. Furthermore, treatment with a PUFA analogue triacsin C (a potent inhibitor of ACSL1, ACSL3 and ACSL4, but not ACSL5) or other inhibitors repressed cancer cell growth in vitro and in vivo (48). Lastly, it was reported that blockage of octamer-binding protein 1 binding to ACSL3 enhancer by the use of pyrrole-imidazole polyamides reduced ACSL3 expression and castration-resistant tumor growth (32). In general, these findings were in line with the observations that ACSLs are upregulated in cancer. However, in a few exceptions, knockdown of ACSL1 or ACSL4 with specific small interfering RNA promoted lung (37) or gastric (25) cancer cell growth, respectively, and in vivo xenograft growth of gastric tumors (25).

ACSLs and deregulated cancer metabolism. It is yet unclear whether the deregulated expression of ACSLs result from the increasing demands of the cancerous tissues to metabolize and store oversupplied lipids. Particularly in those cancer types that originate from tissues with a higher basal level of lipid metabolism and deposition (e.g. liver, colon, breast and prostate cancer), ACSL1 (11-15) and ACSL4 (19-24) were generally upregulated. These two enzymes participate in lipid anabolism and catabolism. By contrast, ACSL5 [another ACSL member which drives lipid biosynthesis rather than fatty acid oxidation (10)] was downregulated in breast and prostate cancer $(26,27)$. It is thus tentative to propose that ACSL1- and ACSL4-mediated lipid catabolism are useful for cancer promotion.

Apart from lipid metabolism, ACSL isoforms can also affect glucose metabolism. ACSL1 overexpression in cancer was found to increase intracellular acylcarnitine and lower basal respiration rates, while ACSL4 upregulation decreased PUFA concentration and promoted glycolysis (11). Combined expression of ACSL1 and ACSL4 together with SCD enabled cells to upregulate phospholipids and urea cycle metabolites. Importantly, the expression level of ACSLs could be altered when cancer cells shift from anabolic lipid metabolism to catabolism. ACSL3 was increased in early carcinogenesis to promote lipid anabolism and deposition, but deceased in advanced breast and prostate cancer to increase lipid utilization $(34,35)$. This shift can promote cancer cell survival and invasiveness, which will be discussed in detail in the following sections.

ACSLs and tissue invasion and metastasis. Cancer cell invasion and migration are characteristic for cancer progression, recurrence and metastasis. Accumulating data have shown that ACSL1 and ACSL4 could promote cancer cell invasion and migration in breast and prostate cancer cells $(20,24,37,43)$. The common epithelial-mesenchymal transition (EMT) contributes to their actions in cell migration and invasion. A series of studies conducted in colon cancer further indicated that ACSL1 and ACSL4 may act synergistically on cancer progression (11). ACSL1 promoted cell invasion, but ACSL4 stimulated cell proliferation and migration in colon cancer cells. The combined overactivation of ACSLs together with SCD contributed to EMT, cancer invasion and poor patient survival in patients with stage II colon cancer (11). Another study also showed that ACSL4-mediated uptake of fatty acids AA and eicosapentaenoic acid was essential for $17 \beta$-estradiol-induced cell migration and invasion in breast cancer cells (43). By contrast, homozygous deletion of ACSL3 promoted distant metastasis in breast cancer patients following adjunct chemotherapy treatment (35). In another investigation 
studying how CUB-domain containing protein 1 mechanically activated high metastatic potency in triple-negative breast cancer cells (49), ACSL3 was again found to decrease invasiveness through increased abundance of lipid droplets and decreased fatty acid oxidation.

Fatty acids are natural ligands of several nuclear transcription factors, e.g., hepatocyte nuclear factor- $4 \alpha$ (HNF- $4 \alpha$ ), PPAR $\alpha$ and retinoic acid X receptor- $\alpha$. Long-chain acyl-CoA thioesters, which are products of ACSL-mediated fatty acid activation, can directly bind to HNF- $4 \alpha$ and modulate its transcriptional activity (50). ACSLs can facilitate HNF-4 $\alpha$ modulation by converting fatty acids to acyl-CoA, while acyl-CoA thioesterase acts in the opposite manner. These modulations are dependent on the chain length and saturation degree of the fatty acyl-CoA thioesters. For example, PUFA $(\alpha$-linolenic acid and eicosatrienoic acid) in the physiological plasma concentration could suppress HNF- $4 \alpha$ transcriptional activity. As HNF- $4 \alpha$ is inversely associated with EMT and $\beta$-catenin signaling activity in liver and colon carcinogenesis (51), the ACSL-mediated modulation of HNF- $4 \alpha$ activity could thus promote cell invasion and migration. Furthermore, ACSL 4 could also upregulate cyclooxygenase-2 (COX-2) expression and synergize with the latter to activate AA metabolism and cancer cell invasion $(47,52)$.

ACSLs and programmed cell death. Apoptosis and other forms of programmed cell death are important for cellular non-immune surveillance to eradicate damaged or mutated cells during carcinogenesis. However, these defensive actions can be utilized smartly by the evolved cancer cells to counteract chemotherapy and other death signals. In general, genetic inhibition of ACSLs could induce lipotoxicity and cell death in cancer cells $(21,23,31,53,54)$. Triacsin $C$ treatment reduced overall ACSL activities and induced cell death in cancer cells, but forced overexpression of the insensitive ACSL5, markedly compensated ACSL activity and rescued cell death induced by triacsin C $(53,55)$. Importantly, the pro-survival function was again replicated in ACSS2 (which utilizes short-chain acetate for fatty acid activation and acetyl-CoA production). ACSS2 is upregulated in numerous types of cancer (e.g., breast and liver cancer, and glioblastoma) and is capable of utilizing acetate as an alternative energy source to support cell survival under metabolic stress (56). Taken together, these results substantiated the role of ACSLs in cancer cell survival.

Fatty acids, particularly AA (a preferred substrate of ACSL4), are apoptosis inducers. This was identified by a landmark study investigating the mechanisms involved in COX-2 inhibition-induced cell death in colon cancer (57). Accumulation of unesterified AA, by either exogenous supplementation in the culture medium or treatment with triacsin C, resulted in cell death. However, in cancer cells, the pro-apoptotic activities of unesterified AA could be neutralized by ACSL4 (which activates AA for esterification into TAG) and COX-2 (which facilitates AA to convert to prostaglandin). Inducible expression of ACSL4 or COX-2 dampened AA accumulation and consequently rescued cell death (57). Note that these two enzymes have been found to be upregulated in colon cancer and other types of cancer, and this study therefore disclosed the oncogenic mechanism of ACSL4 in cancer cell survival.
Unexpectedly, two relevant studies recently reported that ACSL4 was essential for the induction of ferroptosis, another form of programmed cell death. Ferroptosis is characterized by ungoverned lipid peroxidation and it can be prevented by iron chelators and antioxidants $(58,59)$. The first study $(60)$ screened lipid species by the use of redox lipidomics approaches in order to identify potential ferroptosis signals. It was found that the oxidized form of acyl-AA and acyl-adrenic acid (AdA) were necessary and essential for ferroptosis. In the process, ACSL4 facilitated AA and AdA esterification for subsequent lopoxygenase (LOX)-mediated oxidation. Consistently, genetic or pharmacological inhibition of ACSL4 could prevent ferroptosis. The other parallel study (61) utilized two different genetics methodologies to search for essential genes in ferroptosis execution. The clustered regularly interspaced short palindromic repeats-based genetic screening approach and the other transcriptome approach comparing ferroptosis-resistant and -sensitive cells commonly uncovered ACSL4. ACSL4 was then confirmed to be necessary and essential in ferroptosis. These two studies were in line with an earlier report (62) revealing that ACSL4 may mark the sensitivity of ferroptosis in breast cancer cell lines.

Taken together, ACSLs may generally render resistance to fatty acid-induced lipotoxicity and cell death in cancer cells. However, ACSL4 also enables cells to undergo ferroptosis through oxidized AA and AdA. Why ACSL4 directs AA metabolism toward these two distinct pathways (cell survival and ferroptosis) is unresolved. One question to be raised is whether there is a threshold of AA concentration to differentiate survival and ferroptosis. In other words, we propose that ACSL4 is necessary for fatty acid activation; it can cope with a low level of AA metabolism and esterify AA to the COX-2 pathway to promote cell survival. However, excess AA induces Fenton reaction-involved oxidative stress, and the latter may switch off the COX-2 conversion pathway, but turn on the LOX oxidation pathway. Tentative post-translational modifications (probably oxidation of sulfides) of the key amino acids in these two enzyme proteins may affect their activities. Another translational question to be answered is whether ferroptosis inducers can specifically target clinical cancer cells overexpressing ACSL4, but safely spare the non-cancerous tissues with physiological ACSL4 expression in vivo. Considering that ACSL4 is overexpressed in numerous types of cancer, specific activation of ferroptosis may be a desirable anticancer strategy.

\section{Conclusions}

In light of the epidemic of overweight and obese individuals, excess fatty acid metabolism has increasingly been found to be associated with metabolic disorders and carcinogenesis. Based on the evidence examined thus far, ACSL1 and ACSL4 are overexpressed in the majority of cancer types and exhibit oncogenic activities. These ACSLs can promote unchecked cancer cell proliferation, switch on deregulated cancer metabolism, facilitate tumor invasion and metastasis, and evade programmed cell death. ACSL3 is relatively complex, with varying expression and function in the different stages of prostate and breast cancer. By contrast, ACSL5 is generally decreased in those cancer types where ACSL1 and ACSL4 are upregulated. It is thus imperative to clarify the molecular 
mechanisms involved in individual ACSL isoforms and design targeted therapies in a precision mode. Manipulation of ACSL activities by genetic or pharmacological approaches (e.g., triacsin C to inhibit ACSL1 and ACSL4, but spare ACSL5) is theoretically applicable, but the non-specific toxicity cannot be overlooked due to the importance of fatty acid activation in physiology. Notably, ACSL4 marks the sensitivity of ferroptosis, making it a desirable target in cancer. Future studies are necessary to deepen our understanding of ACSL-involved fatty acid metabolism and carcinogenesis.

\section{Acknowledgements}

Not applicable.

\section{Funding}

The present study was supported by the National Natural Science Foundation of China (grant no. 81672370), the Natural Science Foundation of Guangxi Province (grant no. 2015GXNSFCB139004), the Guangxi Hundred-Talent Program (awarded in 2016) and the Guangxi Medical University Training Program for Distinguished Young Scholars (awarded in 2017).

\section{Availability of data and materials}

The datasets used and/or analyzed during the current study are available from the corresponding author on reasonable request.

\section{Authors' contributions}

YT and JZ searched the literature and organized the data. YT drafted the manuscript. SCH provided important suggestions, and critically revised the manuscript. YMJ and GDL designed the research, summarized the data and finalized the paper.

\section{Ethics approval and consent to participate}

Not applicable.

\section{Consent for publication}

Not applicable.

\section{Competing interests}

The authors declare that they have no competing interests.

\section{References}

1. Ellis JM, Frahm JL, Li LO and Coleman RA: Acyl-coenzyme A synthetases in metabolic control. Curr Opin Lipidol 21: 212-217, 2010 .

2. Grevengoed TJ, Klett EL and Coleman RA: Acyl-CoA metabolism and partitioning. Annu Rev Nutr 34: 1-30, 2014.

3. Neess D, Bek S, Engelsby H, Gallego SF and Færgeman NJ: Long-chain acyl-CoA esters in metabolism and signaling: Role of acyl-CoA binding proteins. Prog Lipid Res 59: 1-25, 2015.

4. Digel M, Ehehalt R, Stremmel W and Füllekrug J: Acyl-CoA synthetases: Fatty acid uptake and metabolic channeling. Mol Cell Biochem 326: 23-28, 2009.
5. Fujimoto Y, Itabe H, Kinoshita T, Homma KJ, Onoduka J, Mori M, Yamaguchi S, Makita M, Higashi Y, Yamashita A and Takano T: Involvement of ACSL in local synthesis of neutral lipids in cytoplasmic lipid droplets in human hepatocyte $\mathrm{HuH7}$. J Lipid Res 48: 1280-1292, 2007.

6. Li LO, Ellis JM, Paich HA, Wang S, Gong N, Altshuller G, Thresher RJ, Koves TR, Watkins SM, Muoio DM, et al: Liver-specific loss of long chain acyl-CoA synthetase-1 decreases triacylglycerol synthesis and beta-oxidation and alters phospholipid fatty acid composition. J Biol Chem 284: 27816-27826, 2009.

7. Ellis JM, Li LO, Wu PC, Koves TR, Ilkayeva O, Stevens RD, Watkins SM, Muoio DM and Coleman RA: Adipose acyl-CoA synthetase-1 directs fatty acids toward beta-oxidation and is required for cold thermogenesis. Cell Metab 12: 53-64, 2010.

8. Li LO, Grevengoed TJ, Paul DS, Ilkayeva O, Koves TR, Pascual F, Newgard CB, Muoio DM and Coleman RA: Compartmentalized acyl-CoA metabolism in skeletal muscle regulates systemic glucose homeostasis. Diabetes 64: 23-35, 2015

9. Ellis JM, Mentock SM, Depetrillo MA, Koves TR, Sen S, Watkins SM, Muoio DM, Cline GW, Taegtmeyer H, Shulman GI, et al: Mouse cardiac acyl coenzyme a synthetase 1 deficiency impairs Fatty Acid oxidation and induces cardiac hypertrophy. Mol Cell Biol 31: 1252-1262, 2011.

10. Bowman TA, O'Keeffe KR, D'Aquila T, Yan QW, Griffin JD, Killion EA, Salter DM, Mashek DG, Buhman KK and Greenberg AS: Acyl CoA synthetase 5 (ACSL5) ablation in mice increases energy expenditure and insulin sensitivity and delays fat absorption. Mol Metab 5: 210-220, 2016.

11. Sanchez-Martinez R, Cruz-Gil S, García-Álvarez MS, Reglero G and Ramirez de Molina A: Complementary ACSL isoforms contribute to a non-Warburg advantageous energetic status characterizing invasive colon cancer cells. Sci Rep 7: 11143, 2017.

12. Sánchez-Martínez R, Cruz-Gil S, Gómez de Cedrón M, Álvarez-Fernández M, Vargas T, Molina S, García B, Herranz J, Moreno-Rubio J, Reglero G, et al: A link between lipid metabolism and epithelial-mesenchymal transition provides a target for colon cancer therapy. Oncotarget 6: 38719-38736, 2015.

13. Wang Y, Cai X, Zhang S, Cui M, Liu FSun B, Zhan W and Zhang $X$, and Ye L: HBXIP up-regulates ACSL1 through activating transcriptional factor Sp1 in breast cancer. Biochem Biophys Res Commun 484: 565-571, 2017.

14. Cui M, Wang Y, Sun B, Xiao Z, Ye L and Zhang X: MiR-205 modulates abnormal lipid metabolism of hepatoma cells via targeting acyl-CoA synthetase long-chain family member 1 (ACSL1) mRNA. Biochem Biophys Res Commun 444: 270-275, 2014.

15. Cui M, Xiao Z, Wang Y, Zheng M, Song T, Cai X, Sun B, Ye L and Zhang X: Long noncoding RNA HULC modulates abnormal lipid metabolism in hepatoma cells through an miR-9-mediated RXRA signaling pathway. Cancer research 75: 846-857, 2015.

16. Ivyna Bong PN, Ng CC, Lam KY, Megat Baharuddin PJ, Chang KM and Zakaria Z: Identification of novel pathogenic copy number aberrations in multiple myeloma: The Malaysian. Mol Cytogenet 7: 24, 2014.

17. Huang W, Jin Y, Yuan Y, Bai C, Wu Y2, Zhu H and Lu S: Validation and target gene screening of hsa-miR-205 in lung squamous cell carcinoma. Chin Med J (Engl) 127: 272-278, 2014.

18. Vargas T, Moreno-Rubio J, Herranz J, Cejas P, Molina S, Mendiola M, Burgos E, Custodio AB, De Miguel M et al: 3'UTR polymorphism in ACSL1 gene correlates with expression levels and poor clinical outcome in colon cancer patients. PLoS One 11: e0168423, 2016.

19. Cao Y, Dave KB, Doan TP and Prescott SM: Fatty acid CoA ligase 4 is up-regulated in colon adenocarcinoma. Cancer Res 61: 8429-8434, 2001.

20. Wu X, Li Y, Wang J, Wen X, Marcus MT, Daniels G, Zhang DY, Ye F, Wang LH, Du X, et al: Long chain fatty acyl-CoA synthetase 4 is a biomarker for and mediator of hormone resistance in human breast cancer. PloS one 8: e77060, 2013.

21. Xia H, Lee KW, Chen J, Kong SN, Sekar K, Deivasigamani A, Seshachalam VP, Goh BKP, Ooi LL, Hui KM, et al: Simultaneous silencing of ACSL4 and induction of GADD45B in hepatocellular carcinoma cells amplifies the synergistic therapeutic effect of aspirin and sorafenib. Cell Death Discov 3: 17058, 2017.

22. Sung YK, Hwang SY, Park MK, Bae HI, Kim WH, Kim JC and Kim M: Fatty acid-CoA ligase 4 is overexpressed in human hepatocellular carcinoma. Cancer Sci 94: 421-424, 2003.

23. Hu C, Chen L, Jiang Y, Li Y and Wang S: The effect of fatty acid-CoA ligase 4 on the growth of hepatic cancer cells. Cancer Biol Ther 7: 131-134, 2008. 
24. Wu X, Deng F, Li Y, Daniels G, Du X, Ren Q, Wang J, Wang LH, Yang Y, Zhang V, et al: ACSL4 promotes prostate cancer growth, invasion and hormonal resistance. Oncotarget 6: 44849-44863, 2015.

25. Ye X, Zhang Y, Wang X, Li Y and Gao Y: Tumor-suppressive functions of long-chain acyl-CoA synthetase 4 in gastric cancer. IUBMB Life 68: 320-327, 2016.

26. Gassler N, Schneider A, Kopitz J, Schnölzer M, Obermüller N, Kartenbeck J, Otto HF and Autschbach F: Impaired expression of acyl-CoA-synthetase 5 in epithelial tumors of the small intestine. Hum Pathol 34: 1048-1052, 2003.

27. Yen MC, Kan JY, Hsieh CJ, Kuo PL, Hou MF and Hsu YL: Association of long-chain acyl-coenzyme A synthetase 5 expression in human breast cancer by estrogen receptor status and its clinical significance. Oncol Rep 37: 3253-3260, 2017.

28. Gaisa NT, Reinartz A, Schneider U, Klaus C, Heidenreich A Jakse G, Kaemmerer E, Klinkhammer BM, Knuechel R and Gassler N: Levels of acyl-coenzyme A synthetase 5 in urothelial cells and corresponding neoplasias reflect cellular differentiation. Histol Histopathol 28: 353-364, 2013.

29. Yamashita Y, Kumabe T, Cho YY, Watanabe M, Kawagishi J, Yoshimoto T, Fujino T, Kang MJ and Yamamoto TT: Fatty acid induced glioma cell growth is mediated by the acyl-CoA synthetase 5 gene located on chromosome 10q25.1-q25.2, a region frequently deleted in malignant gliomas. Oncogene 19: 5919-5925, 2000.

30. Kim SY, Ahn T, Bang H, Ham JS, Kim J, Kim ST, Jang J, Shim M, Kang SY, Park SH, et al: Acquired resistance to LY2874455 in FGFR2-amplified gastric cancer through an emergence of novel FGFR2-ACSL5 fusion. Oncotarget 8: 15014-15022, 2017.

31. Padanad MS, Konstantinidou G, Venkateswaran N, Melegari M, Rindhe S, Mitsche M, Yang C, Batten K, Huffman KE, Liu J, et al: Fatty acid oxidation mediated by acyl-CoA synthetase long Chain 3 is required for mutant KRAS lung tumorigenesis. Cell Rep 16: 1614-1628, 2016.

32. Obinata D, Takayama K, Fujiwara K, Suzuki T, Tsutsumi S, FukudaN,NagaseH,FujimuraT,UranoT,Homma Y,etal:Targeting Oct1 genomic function inhibits androgen receptor signaling and castration-resistant prostate cancer growth. Oncogene 35 : 6350-6358, 2016.

33. Wang J, Scholtens D, Holko M, Ivancic D, Lee O, $\mathrm{Hu} \mathrm{H}$, Chatterton RT Jr, Sullivan ME, Hansen N, Bethke K, et al: Lipid metabolism genes in contralateral unaffected breast and estrogen receptor status of breast cancer. Cancer Prev Res (Phila) 6 : 321-330, 2013

34. Marques RB, Dits NF, Erkens-Schulze S, van Ijcken WF, van Weerden WM and Jenster G: Modulation of androgen receptor signaling in hormonal therapy-resistant prostate cancer cell lines. PLoS One 6: e23144, 2011.

35. Jeong HM, Kim RN, Kwon MJ, Oh E, Han J, Lee SK, Choi JS Park S, Nam SJ, Gong GY, et al: Targeted exome sequencing of Korean triple-negative breast cancer reveals homozygous deletions associated with poor prognosis of adjuvant chemotherapy-treated patients. Oncotarget 8: 61538-61550, 2017

36. Su RJ, Jonas BA, Welborn J, Gregg JP and Chen M: Chronic eosinophilic leukemia, NOS with t(5;12)(q31;p13)/ETV6-ACSL6 gene fusion: A novel variant of myeloid proliferative neoplasm with eosinophilia. Hum Pathol (N Y) 5: 6-9, 2016.

37. Chen WC, Wang CY, Hung YH, Weng TY, Yen MC and Lai MD: Systematic analysis of gene expression alterations and clinical outcomes for long-chain acyl-coenzyme a synthetase family in cancer. PLoS One 11: e0155660, 2016.

38. Weedon-Fekjaer MS, Dalen KT, Solaas K, Staff AC, Duttaroy AK and Nebb HI: Activation of LXR increases acyl-CoA synthetase activity through direct regulation of ACSL3 in human placental trophoblast cells. J Lipid Res 51: 1886-1896, 2010.

39. Cao A, Li H, Zhou Y, Wu M and Liu J: Long chain acyl-CoA synthetase-3 is a molecular target for peroxisome proliferator-activated receptor delta in HepG2 hepatoma cells. J Biol Chem 285: 16664-16674, 2010

40. Chang YS, Tsai CT, Huangfu CA, Huang WY, Lei HY, Lin CF, Su IJ, Chang WT, Wu PH, Chen YT, et al: ACSL3 and GSK-3 $\beta$ are essential for lipid upregulation induced by endoplasmic reticulum stress in liver cells. J Cell Biochem 112: 881-893, 2011

41. Cui M, Xiao Z, Sun B, Wang Y, Zheng M, Ye L and Zhang X: Involvement of cholesterol in hepatitis $B$ virus $X$ protein-induced abnormal lipid metabolism of hepatoma cells via up-regulating miR-205-targeted ACSL4. Biochem Biophys Res Commun 445: $651-655,2014$
42. Kan CF, Singh AB, Stafforini DM, Azhar S and Liu J: Arachidonic acid downregulates acyl-CoA synthetase 4 expression by promoting its ubiquitination and proteasomal degradation. J Lipid Res 55: 1657-1667, 2014.

43. Belkaid A, Ouellette RJ and Surette ME: $17 \beta$-estradiol-induced ACSL4 protein expression promotes an invasive phenotype in estrogen receptor positive mammary carcinoma cells. Carcinogenesis 38: 402-410, 2017.

44. Yao $\mathrm{H}$ and Ye J: Long chain acyl-CoA synthetase 3-mediated phosphatidylcholine synthesis is required for assembly of very low density lipoproteins in human hepatoma Huh7 cells. J Biol Chem 283: 849-854, 2008.

45. Hanahan D and Weinberg RA: Hallmarks of cancer: The next generation. Cell 144: 646-674, 2011.

46. Migita T, Takayama KI, Urano T, Obinata D, Ikeda K, Soga T, Takahashi S and Inoue S: ACSL3 promotes intratumoral steroidogenesis in prostate cancer cells. Cancer Sci 108: 2011-2021, 2017.

47. Orlando UD, Garona J, Ripoll GV, Maloberti PM, Solano AR, Avagnina A, Gomez DE, Alonso DF and Podestá EJ: The functional interaction between acyl-CoA synthetase 4, 5-lipooxygenase and cyclooxygenase- 2 controls tumor growth: A novel therapeutic target. PLoS One 7: e40794, 2012.

48. Orlando UD, Castillo AF, Dattilo MA, Solano AR, Maloberti PM and Podesta EJ: Acyl-CoA synthetase-4, a new regulator of mTOR and a potential therapeutic target for enhanced estrogen receptor function in receptor-positive and -negative breast cancer. Oncotarget 6: 42632-42650, 2015.

49. Wright HJ, Hou J, Xu B, Cortez M, Potma EO, Tromberg BJ and Razorenova OV: CDCP1 drives triple-negative breast cancer metastasis through reduction of lipid-droplet abundance and stimulation of fatty acid oxidation. Proc Natl Acad Sci USA 114: E6556-E6565, 2017.

50. Hertz R, Magenheim J, Berman I and Bar-Tana J: Fatty acyl-CoA thioesters are ligands of hepatic nuclear factor-4alpha. Nature 392: 512-516, 1998.

51. Ning BF, Ding J, Yin C, Zhong W, Wu K, Zeng X, Yang W, Chen YX, Zhang JP and Zhang X: Hepatocyte nuclear factor 4 alpha suppresses the development of hepatocellular carcinoma. Cancer Res 70: 7640-7651, 2010.

52. Maloberti PM, Duarte AB, Orlando UD, Pasqualini ME, Solano AR, López-Otín C and Podestá EJ: Functional interaction between acyl-CoA synthetase 4, lipooxygenases and cyclooxygenase-2 in the aggressive phenotype of breast cancer cells. PLoS One 5: e15540, 2010

53. Mashima T, Sato S, Okabe S, Miyata S, Matsuura M, Sugimoto Y, Tsuruo T and Seimiya H: Acyl-CoA synthetase as a cancer survival factor: Its inhibition enhances the efficacy of etoposide. Cancer Sci 100: 1556-1562, 2009.

54. Mashima T, Sato S, Sugimoto Y, Tsuruo T and Seimiya H: Promotion of glioma cell survival by acyl-CoA synthetase 5 under extracellular acidosis conditions. Oncogene 28: 9-19, 2009.

55. Mashima T, Oh-hara T, Sato S, Mochizuki M, Sugimoto Y, Yamazaki K, Hamada J, Tada M, Moriuchi T, Ishikawa Y, Kato Y, et al: p53-defective tumors with a functional apoptosome-mediated pathway: A new therapeutic target. J Natl Cancer Inst 97: 765-777, 2005.

56. Schug ZT, Peck B, Jones DT, Zhang Q, Grosskurth S, Alam IS, Goodwin LM, Smethurst E, Mason S1, Blyth K, et al: Acetyl-CoA synthetase 2 promotes acetate utilization and maintains cancer cell growth under metabolic stress. Cancer Cell 27: 57-71, 2015.

57. Cao Y, Pearman AT, Zimmerman GA, McIntyre TM and Prescott SM: Intracellular unesterified arachidonic acid signals apoptosis. Proc Natl Acad Sci USA 97: 11280-11285, 2000.

58. Stockwell BR, Friedmann Angeli JP, Bayir H, Bush AI, Conrad M, Dixon SJ, Fulda S, Gascón S, Hatzios SK and Kagan VE: Ferroptosis: A regulated cell death nexus linking metabolism, redox biology, and disease. Cell 171: 273-285, 2017.

59. Yang WS and Stockwell BR: Ferroptosis: Death by lipid peroxidation. Trends Cell Biol 26: 165-176, 2016.

60. Kagan VE, Mao GW, Qu F, Angeli JP, Doll S, Croix CS, Dar HH, Liu B, Tyurin VA, Ritov VB, et al: Oxidized arachidonic and adrenic PEs navigate cells to ferroptosis. Nat Chem Biol 13: 81-90, 2017.

61. Doll S, Proneth B, Tyurina YY, Panzilius E, Kobayashi S, Ingold I, Irmler M, Beckers J, Aichler M, Walch A, et al: ACSL4 dictates ferroptosis sensitivity by shaping cellular lipid composition. Nat Chem Biol 13: 91-98, 2017.

62. Yuan H, Li X, Zhang X, Kang R and Tang D: Identification of ACSL4 as a biomarker and contributor of ferroptosis. Biochem Biophys Res Commun 478: 1338-1343, 2016. 